\title{
Prostate Specific Antigen Serum Levels in Patients with Different Levels of Hepatic or Renal Impairment and in Those with Systemic Inflammation in a University Hospital. A Retrospective Analysis of 10 Years of Laboratory Data
}

\author{
Toth $\mathrm{Z}^{1}$, Szalay B ${ }^{2}$, Gyarmati B ${ }^{3}$, Jalal DA², Vasarhelyi B ${ }^{2,4 *}$ and Szabo $\mathrm{T}^{2}$ \\ ${ }^{1}$ Department of Urology, Uzsoki Hospital, Hungary \\ ${ }^{2}$ Department of Laboratory Medicine, Semmelweis University, Hungary \\ ${ }^{3}$ Department of Gynecology and Obstetrics, Uzsoki Hospital, Hungary \\ ${ }^{4}$ Hungarian Academy of Sciences, Hungary
}

*Corresponding author: Barna Vasarhelyi, Department of Laboratory Medicine, Semmelweis University, Hungary, Tel: +36206663246; Email: vasarhelyi.barna@med.semmelweis-univ.hu

\section{Research Article \\ Volume 5 Issue 3}

Received Date: September 07, 2020

Published Date: October 05, 2020

DOI: $10.23880 /$ oajun-16000184

\section{Abstract}

Introduction: Prostate specific antigen (PSA) level is widely used as a screening tool for the identification of patients with increased risk of prostate malignancy. We tested whether PSA levels are affected by liver or renal impairment or systemic inflammation.

Methods: We performed a retrospective analysis of almost 200 million records of the last 10 years of laboratory data. We selected those patients having both PSA and any of alanine aminotransferase (ALT), estimated glomerular filtration rate (eGFR) or C-reactive protein (CRP) results. We calculated the 5, 25, 50 (median), 75 and 95 per centile PSA values. Then the associations were adjusted for patients' age.

Results: We found significant association between ALT, eGFR and CRP levels and PSA. When age was included in the analysis the association between ALT or eGFR and PSA disappeared. The impact of PSA on CRP remained significant after its adjustment to patients' age. Median and 75 per centile PSA values were more than $20 \%$ and $50 \%$ higher in patients with severe systemic inflammation (defined as CRP levels $>50 \mathrm{mg} / \mathrm{L}$ ) compared to those with low CRP (CRP levels $<5 \mathrm{mg} / \mathrm{L}$ ), respectively.

Discussion: Reference and cut-off values of PSA should be adjusted just for patients' age without considering their hepatic or renal condition. Elevated PSA levels should be interpreted cautiously in patients with severe inflammation. It is recommended to postpone PSA-based screening until the normalization of CRP.

Keywords: Prostate Malignancy; Retrospective Analysis; Renal Condition; Prostate Cancer; Renal Disease

Abbreviations: PSA: Prostate Specific Antigen; eGFR: estimated Glomerular Filtration Rate; CRP: C-Reactive Protein; ALT: Alanine Aminotransferase.

\section{Introduction}

Prostate specific antigen (PSA) level is widely used as a screening tool for the identification of patients with 


\section{Open Access Journal of Urology \& Nephrology}

increased risk of prostate malignancy for decades [1] as with any other laboratory parameter any change of PSA cut-off values has a profound effect on laboratory-based screening sensitivity and specificity values [2]. currently, the commonly used threshold value for clinical decision making is $4 \mu \mathrm{g} / \mathrm{L}$, but this value is not routinely adjusted to extra-prostate factors. Some of these extra-prostate factors as age are widely known, while others such as thyroid function, daily or seasonal variations are identified more recently $[3,4]$.

As prostate cancer is the characteristic disease of ageing population it is not uncommon that it affects patients with simultaneous presence of chronic conditions. Hepatic and renal disease and low level systemic inflammation are characteristic abnormalities that affect a significant part of patients. These conditions are, however, may also have an impact on synthesis, release, metabolism, composition and lifetime of different laboratory analytes include specific proteins. The retrospective analysis of laboratory test results provides a powerful approach to elucidate the interaction of different laboratory parameters. In our study we tested the distributions of PSA levels in our laboratory database in patients with hepatic or renal impairment or with systemic inflammation. The conditions were defined according to laboratory abnormalities.

\section{Methods}

The Department of Laboratory Medicine provides general laboratory services for 44 University Hospitals of Semmelweis University, Budapest, Hungary. From Laboratory Informatics System we retrieved records generated since $1^{\text {st }}$ January, 2011 that included the following fields: anonymized patient identification number; gender; age; date of measurement; the name of the measured parameter; test result; reference range and unit; and instrument used for testing. Out of the collected $\approx 200$ million records we selected those that fulfilled the following criteria:

- Male gender

- Any of estimated glomerular filtration rate (eGFR) or alanine transaminase (ALT) or C-reactive protein (CRP) levels

- PSA levels
All laboratory parameters were measured by CE IVD qualified, commercially available tests.

In order to exclude patients with severe prostate pathology we arbitrarily excluded records with PSA levels above $20 \mu \mathrm{g} / \mathrm{L}$ from further analysis. Then we selected those patients having both PSA AND any of the listed parameters within 30 days. From the created database we selected and used for further analysis those data pairs for individual patients that were measured for the first time in our database. We generated subgroups and cohorts according to ALT, eGFR, and CRP and calculated the 5, 25, 50 (median), 75 and 95 per centile PSA values.

In addition, the independent effect of eGFR, ALT and CRP and age on PSA levels was determined with logistic regression analysis of logarithmic data. (Statistical analysis was performed with R software package.) The data analysis was approved by an Independent Ethical Committee of the University. To test the impact of laboratory abnormalities on PSA levels we performed a series of logistic regression analyses with different analytes and age as independent and PSA as dependent variables. We also tested the direct association between age and individual analytes. For the analyses, logarithmic transformation of PSA and laboratory parameters was performed in order to achieve a close-tonormal distribution in individual dimensions. Due to the characteristics of the PSA test, any value measured to be below $0.1 \mu \mathrm{g} / \mathrm{l}$ is reported here as $0.1 \mathrm{ug} / \mathrm{l}$.

\section{Results}

Table 1 summarizes the results of our analysis. Median PSA values were seemingly lower in patients with high ALT and CRP and low eGFR levels. We found significant association between ALT, eGFR and CRP levels and PSA. When age was included in the analysis the association between ALT or eGFR and PSA disappeared. High CRP levels, however, remained significant predictors for high PSA levels. For estimates, standard error and probability values, please, refer to Table 2 .

\begin{tabular}{|c|c|c|c|c|c|c|c|}
\hline \multirow{2}{*}{ Label } & \multirow{2}{*}{ Case Number } & \multirow{2}{*}{$\begin{array}{c}\text { Patients' mean } \\
\text { age }\end{array}$} & \multicolumn{5}{|c|}{ Per centile value } \\
\hline & & & 5 & 25 & 50 (median) & 75 & 95 \\
\hline \multicolumn{8}{|c|}{ ALT, IU/L } \\
\hline$<40$ & 19887 & 61.5 & 0.1 & 0.54 & 1 & 2.16 & 10.94 \\
\hline $40-<80$ & 3675 & 55.3 & 0.1 & 0.47 & 0.83 & 1.6 & 8.71 \\
\hline $80-120$ & 653 & 55.5 & 0.1 & 0.43 & 0.77 & 1.4 & 7.79 \\
\hline$>120$ & 552 & 57.2 & 0.1 & 0.4 & 0.73 & 1.61 & 9.92 \\
\hline
\end{tabular}




\begin{tabular}{|c|c|c|c|c|c|c|c|}
\hline \multicolumn{8}{|c|}{$\mathrm{eGFR} \mathrm{ml} / \mathrm{min} / 1,73 \mathrm{~m}^{2}$} \\
\hline$<15$ & 296 & 62.3 & 0.1 & 0.67 & 1.12 & 2.77 & 13.43 \\
\hline $15-<30$ & 596 & 68.8 & 0.1 & 0.58 & 1.3 & 3.14 & 12.57 \\
\hline $30-<60$ & 4412 & 68.5 & 0.1 & 0.61 & 1.26 & 2.94 & 12.85 \\
\hline $60-90$ & 14146 & 60.5 & 0.1 & 0.52 & 0.96 & 2.05 & 10.37 \\
\hline$>90$ & 4743 & 52.4 & 0.1 & 0.48 & 0.8 & 1.43 & 6.97 \\
\hline \multicolumn{8}{|c|}{ CRP, mg/L } \\
\hline$<5$ & 11487 & 58 & 0.1 & 0.53 & 0.92 & 1.8 & 9.04 \\
\hline $5-<10$ & 2401 & 61.8 & 0.1 & 0.5 & 0.97 & 2.1 & 10.12 \\
\hline $10-<20$ & 1786 & 64.5 & 0.1 & 0.52 & 1.03 & 2.39 & 12.17 \\
\hline $20-50$ & 1740 & 66 & 0.1 & 0.49 & 1.08 & 2.73 & 11.99 \\
\hline$>50$ & 2238 & 66.9 & 0.1 & 0.48 & 1.16 & 2.9 & 14.25 \\
\hline
\end{tabular}

Table 1: Prostate specific antigen per centile values in patients with different stages of laboratory abnormalities a retrospective descriptive data analysis of laboratory test results measured at Semmelweis University since $1^{\text {st } J a n u a r y, ~} 2011$.

\begin{tabular}{|c|c|c|c|}
\hline Coefficients: & Estimate & Std.error & P value \\
\hline \multicolumn{5}{|c|}{ when age was NOT included in the analysis } \\
\hline $\log$ ALT & -0.125 & 0.013 & $<2 \mathrm{e}-16^{*}$ \\
\hline $\log$ eGFR & -0.147 & 0.024 & $1.12 \mathrm{e}-09^{*}$ \\
\hline $\log$ CRP & 0.024 & 0.005 & $9.70 \mathrm{e}-06^{*}$ \\
\hline \multicolumn{7}{|c|}{ when age is included in the analysis } \\
\hline age & 0.0088 & 0.0003 & $<2 \mathrm{e}-16^{*}$ \\
\hline $\log$ ALT & -0.0068 & 0.014 & 0.642 \\
\hline $\log$ eGFR & -0.0459 & 0.025 & 0.076 \\
\hline $\log$ CRP & -0.0335 & 0.011 & $0.002^{*}$ \\
\hline $\log$ ALT & association between age AND & $<2 \mathrm{e}-16^{*}$ \\
\hline $\log$ eGFR & -0.025 & 0.002 & $<2 \mathrm{e}-16^{*}$ \\
\hline $\log$ CRP & -0.194 & 0.006 & $<2 \mathrm{e}-16^{*}$ \\
\hline
\end{tabular}

Table 2: Results of logistic regression analysis.

\section{Discussion}

In our retrospective data analysis we tested the hypothesis that reference ranges should be modified in the presence of some common laboratory abnormalities indicating the impairment of liver and hepatic function and/or the presence of systemic inflammation. For almost 3 decades the measurement of PSA levels is an essential part of laboratory armamentarium aiming to detect prostate cancer early. With ten millions of tests performed worldwide annually the significance of the adequate determination of PSA cut-off values for clinical decision making cannot be overestimated. The use of an inappropriately low cut- off value would lead to an increase of false positive results associated with a dramatic increase of unsubstantiated investigations, while an inappropriately high cut-off value would lead to a high rate of false-negative results and increased rate of undetected prostate cancer cases. PSA values are largely affected by patients' age, as PSA levels are increasing per se in the ageing man without overt prostate pathology. Therefore, it is recommended to apply age-specific reference ranges in order to increase screening specificity [5]. The ageing population, however, is at an increased risk for hepatic and renal impairment and, also, is commonly exposed to chronic low-grade systemic inflammation. Each of these abnormalities can be associated with an alteration 


\section{Open Access Journal of Urology \& Nephrology}

of PSA synthesis, release, composition (relative rate of PSA bound to proteins) and lifetime. Indeed, several reports provided data on the impact of hepatic and renal impairment and systemic inflammation on PSA levels.

Vicentini, et al. [6] analysed PSA levels in hepatic cirrhosis patients. This study prospectively evaluated patients with severe hepatic impairment on waiting list for liver transplantation and reported an inverse relationship between the severity of fibrosis and levels PSA. Inci, et al. [7] reported similar observations in liver cirrhosis patients grouped according to Child-Pugh score. These data obtained in relatively small number of subjects $(n=112$ and 82, respectively) are in line with those of two large epidemiological studies 16563 men with different levels of non-fatty liver disease and 6705 men with different levels of liver fibrosis $[8,9]$. Both of these studies revealed a significantly decreased likelihood of having an abnormal PSA level in patients with advanced liver disease. Several group investigated PSA-levels in patients with end-stage kidney disease with or without dialysis [10-12] and in those with different levels of kidney impairment [13]. Results indicate that total PSA levels are not affected significantly by kidney function per se and renal function has no impact on total PSA levels. Therefore, the reference ranges for total PSA levels should not be adjusted to the severity of renal impairment $[14,15]$.

CRP is the widely accepted marker of systemic inflammation. Several studies suggest that, additionally, it can be also used as a prognostic factor in prostate cancer [16-19]. Two studies performed on healthy men indicated, however, that while neutrophil count and ratio and fibrinogen levels are directly related to PSA, CRP levels themselves have no association with PSA $[20,21]$. These studies were performed on populations with well characterized health status and / or on patients enrolled according to specific characteristics. The situation, however, is not necessarily the same in everyday clinical practice where clinicians should make their decision on a mixed ageing patient population suffering in different chronic and acute conditions. Data collected from such a population, therefore, would be invaluable as they would reflect 'real-life' situation.

In our retrospective analysis around 20,000 patients' PSA values and laboratory results were processed. A weakness of our analysis was that we had access just to patients' age and laboratory data without diagnosis and medical history. Our results were still in line with published reports: patients with hepatic or kidney impairment (defined according to laboratory abnormalities) were presented with lower or higher than average PSA levels, respectively. However, these associations disappeared when patients' age was also considered suggesting, that the impact of these conditions alone is negligible on PSA levels. (An extension of our analysis revealed that age itself is associated with high ALT and low eGFR values). Therefore, the cut-off values of PSA levels should not be adjusted for ALT or eGFR. We should emphasize, however, that we were not aware of patients' diagnosis and overall clinical condition. We cannot exclude the possibility that there could be some specific hepatic or renal disorders having a significant impact on PSA metabolism or lifetime.

In contrast with ALT or eGFR, CRP levels are directly associated with higher PSA levels. Median and 75 per centile PSA values were more than $20 \%$ and $50 \%$ higher in patients with severe systemic inflammation (defined as CRP levels $>50 \mathrm{mg} / \mathrm{L}$ ) compared to those with low CRP (CRP levels $<5$ $\mathrm{mg} / \mathrm{L}$ ), respectively. This association remained significant after its adjustment to patients' age. Based on this finding, therefore, it is recommended to postpone PSA-based screening until the normalization of CRP.

\section{Conclusion}

In summary, our analysis, therefore, reassures interested readers that reference and cut-off values of PSA should be adjusted just for patients' age without considering their hepatic or renal condition in general, while elevated PSA levels should be interpreted cautiously in patients with severe inflammation.

\section{Acknowledgement}

This work was supported by Grant No. GINOP-2.2.1-15-2017-00067.

\section{References}

1. Catalona WJ, Smith DS, Ratliff TL, Dodds KM, Coplen DE, et al. (1991) Measurement of prostate-specific antigen in serum as a screening test for prostate cancer. N Engl J Med 324(17): 1156-1161.

2. Hoffmann RM: Screening for prostate cancer UpToDate ${ }^{\circledR}$ 2020, Aug.

3. Connolly D, van Leeuwen PJ, Bailie J, Black A, Murray LJ, et al. (2011) Daily, monthly and seasonal variation in PSA levels and the association with weather parameters. Prostate Cancer Prostatic Dis 14(1): 58-62

4. Tóth Z, Gyarmati B, Szabó T, Vásárhelyi B (2019) An inverse significant association between thyroid stimulatory hormone (TSH) and prostate specific antigen (PSA) blood levels in males 40-75 years of age 40-75 years of age. Orv Hetil 160(35): 1376-1379.

5. Partin AW, Criley SR, Subong EN, Zincke H, Walsh PC, et al. (1996) Standard versus age-specific prostate specific 


\section{Open Access Journal of Urology \& Nephrology}

antigen reference ranges among men with clinically localized prostate cancer: A pathological analysis. J Urol 155(4): 1336-1339.

6. Vicentini FC, Botelho LA, Hisano M, Ebaid GX, Lucon M, et al. (2009) Are total prostate-specific antigen serum levels in cirrhotic men different from those in normal men?. Urology 73(5): 1032-1035.

7. Inci M, Rifaioglu MM, Inci M, Celik M, Demir M, et al. (2013) The investigation of total PSA, free PSA, and free/ total PSA ratio in patients with liver cirrhosis patients according to Child-Pugh score. Urology 81(3): 617-622.

8. Yoon JH, Yang HJ, Kim JH, Doo SW, Yang WJ, et al. (2015) The likelihood of having a serum PSA level of $\geq 2.5 \mathrm{ng} /$ $\mathrm{mL}$ according to the degree of fatty liver disease in a screened population Can Urol Assoc J 9(11-12): 868872.

9. Wang A, Lazo M, Carter HB, Groopman JD, Nelson WG, et al. (2019) Association between Liver Fibrosis and Serum PSA among U.S. Men: National Health and Nutrition Examination Survey (NHANES), 2001-2010. Cancer Epidemiol Biomarkers Prev 28(8): 1331-1338.

10. Sasagawa I, Kubota Y, Hayami S, Adachi M, Nakada T, et al. (1998) Serum levels of total and free prostate specific antigen in men on hemodialysis. J Urol 160(1): 83-85.

11. Danişman A, Kiliç S, Kukul E, Yakupoğlu G, Güntekin E, et al. (2000) Do renal failure and hemodialysis have any effect on the elimination of free and total prostatespecific antigen?. Eur Urol 37(5): 579-581.

12. Douville P, Tiberi M (1998) Effect of terminal renal failure on the ratio of free to total prostate-specific antigen. Tumour Biol 19(2): 113-117.

13. Bruun L, Savage C, Cronin AM, Hugosson J, Lilja H, et al. (2009) Increase in percent free prostate-specific antigen in men with chronic kidney disease. Nephrol Dial
Transplant 24(4): 1238-1241.

14. Amiri FA (2016) Serum tumor markers in chronic kidney disease: as clinical tool in diagnosis, treatment and prognosis of cancers. Ren Fail 38(4): 530-544.

15. Coppolino G, Bolignano D, Rivoli L, Mazza G, Presta P, et al. (2014) Tumour markers and kidney function: a systematic review. Bio Med Res Int article ID: 647541.

16. Lehrer S, Diamond EJ, Mamkine B, Droller MJ, Stone NN, et al. (2005) C-reactive protein is significantly associated with prostate-specific antigen and metastatic disease in prostate cancer. BJU Int 95(7): 961-962.

17. Beer TM, Lalani AS, Lee S, Mori M, Eilers KM, et al. (2008) ASCENT Investigators. C-reactive protein as a prognostic marker for men with androgen-independent prostate cancer: results from the ASCENT trial. Cancer 112: 2377 2383.

18. Kim Y, Jeon Y, Lee H, Lee D, Shim B (2013) The prostate cancer patient had higher C-reactive protein than BPH patient. Korean J Urol 54(2): 85-88.

19. Prins RC, Rademacher BL, Mongoue-Tchokote $S$, Alumkal JJ, Graff JN, et al. (2012) C-reactive protein as an adverse prognostic marker for men with castration-resistant prostate cancer (CRPC): confirmatory results. Urol Oncol 30(1): 33-37.

20. Yun J, Lee H, Yang W (2017) Association between systemic inflammation and serum prostate-specific antigen in a healthy Korean population. Turk J Urol 43(3): 284-288.

21. McDonald AC, Vira MA, Vidal AC, Gan W, Freedland SJ, et al. (2014) Association between systemic inflammatory markers and serum prostate-specific antigen in men without prostatic disease - the 2001-2008 National Health and Nutrition Examination Survey. Prostate 74(5): 561-567. 